\title{
Liver Cancer Survival: A Real World Observation of 45 Years with 32,556 Cases
}

\author{
Jian-Guo Chen (iD) 1,2 \\ Jian Zhu' \\ Yong-Hui Zhang' \\ Yong-Sheng Chen' \\ Lu-Lu Ding ' \\ Hai-Zhen Chen ${ }^{2}$ \\ Ai-Guo Shen ${ }^{2}$ \\ Gao-Ren Wang ${ }^{2}$ \\ 'Department of Epidemiology, Qidong \\ Liver Cancer Institute, Qidong People's \\ Hospital, Affiliated Qidong Hospital of \\ Nantong University, Qidong, People's \\ Republic of China; ${ }^{2}$ Cancer Institute, \\ Nantong Tumor Hospital, Affiliated \\ Tumor Hospital of Nantong University, \\ Nantong, People's Republic of China
}

Correspondence: Jian-Guo Chen Qidong Liver Cancer Institute, Qidong People's Hospital, Affiliated Qidong Hospital of Nantong University; Cancer Institute, Affiliated Tumor Hospital of Nantong University, Nantong, People's Republic of China

Email chenjg@ntu.edu.cn
Background and Aims: To explore the long-term trend of liver cancer survival, based on the real-world data (RWD) in the past 45 years from a population-based cancer registry, in Qidong, China.

Methods: A number of 32,556 patients with liver cancer were registered during the period of 1972 to 2016. Mixed methods by active and passive follow-up were performed. Life table method was employed for survival analysis by SPSS22 software. Wilcoxon (Gehan) statistics was considered as a significant test. Relative survival was calculated by using SURV software, and its annual percent change (APC) was estimated by the Joinpoint Regression Program.

Results: The overall observed survival (OS) rates of 1-, 5-, 10-, and 20-year rates from the data series were $18.51 \%, 6.28 \%, 4.03 \%$, and $2.84 \%$, and their relative survival (RS) rates were $18.88 \%, 6.95 \%, 4.96 \%$, and $4.49 \%$, respectively. For 24,338 male cases, the 5 -year OS and RS rates were $5.93 \%$ and $6.54 \%$, and for 8218 female cases, $7.34 \%$ and $8.15 \%$, respectively, with $P$ values less than 0.01 . Survival rates of liver cancer from three 15 -year periods of 1972-1986, 1987-2001, and 2002-2016 have increased significantly, with 5-year OS rates of $2.02 \%, 4.40 \%$, and $10.76 \%$, 5-year RS rates of $2.18 \%, 4.83 \%$, and $12.18 \%$; 10 year OS and RS rates of $0.95 \%, 3.00 \%$, and $7.02 \%$, vs $1.13 \%, 3.65 \%$, and $8.96 \%$, respectively, showing a very significant upward trend $(P<0.01)$. There are significant differences among age groups $(P<0.01)$ : those aged 55-64 demonstrated the best OS and RS rates of 5-year, being $8.44 \%$ and $9.09 \%$, respectively.

Conclusion: There are significant gender and age differences in the survival rate of liver cancer in Qidong. RWD indicates the relative lower survival rate of liver cancer in this area, but great improvement has been achieved over the past decades.

Keywords: liver cancer, survival rate, long-term trend, real-world data, Qidong

\section{Background}

Primary liver cancer (PLC) is the sixth most commonly diagnosed cancer and the third leading cause of cancer death with estimated 905,677 new cases and 830,180 deaths worldwide in 2020. The highest rates were observed mainly in Eastern and South- Eastern Asia, and Northern and Western Africa. ${ }^{1}$ In China, liver cancer is the second leading cause of cancer death for decades; about 326,000 deaths a year, with a crude rate of 23.72 per $100,000\left(/ 10^{5}\right)$, and an age-standardized rate of 15.09 / $10^{5}$ in $2015,^{2}$ for instance. Studies in the past decades have shown that the burden of liver cancer in China is characterized by significant geographic distribution, that is, the rates of incidence and mortality of liver cancer in the Eastern region, such as the middle and lower reaches of the Yangtze River, were significantly higher than 
that in the Middle and Western regions in China. ${ }^{3}$ Qidong city, Jiangsu province, is one of the high-rate area among the region, with very high liver cancer mortality rates during the past 60 years: The observed crude rate of liver cancer increased from $20.45 / 10^{5}$ in the 1950's to about $50 / 10^{5}$ in the middle 1970 's, and reached to the highest level of more than $80 / 10^{5}$ at the turn of this century. ${ }^{3}$ Due to the high mortality rate and the poor prognosis, liver cancer constituted a serious disease burden in this area.

Survival analysis is an index used commonly for evaluating the effect (outcome) of treatment of patients in specific settings. There have been numerous reports on liver cancer survival revealing improvements through various treatment, derived from such as therapy-based series, ${ }^{4-7}$ or hospital-based datasets. ${ }^{8-10}$ Recent years, population-based cancer survival has drawn great attentions globally, such as reports from studies in developing countries, EUROCARE studies, or CONCORD series. These research reports have provided very useful information on the general efficiency of cancer health services, based on data from many countries or areas, reflecting a period results for a region, a continent, or five continents. ${ }^{11-14}$

Recent years, within the oncology community, growing interest exists in using data from the real world to address clinical and policy-relevant questions, hence resulting in increased reporting of studies using real world data (RWD). ${ }^{15}$ However, there are few reports derived from long-run population-based cancer registration systems for over decades. In this article, we use data collected in Qidong Cancer Registry (Department of Epidemiology) of Qidong Liver Cancer Institute from a real world observation of 45 year period (1972-2016) to analyze all covered patients with PLC, for delineating of survival status in an area with heavy burden of this disease.

\section{Materials and Methods}

\section{Patient Source}

The Qidong Cancer Registry (QCR), a population-based cancer registry was established in 1972 when the Qidong Liver Cancer Institute was run, ${ }^{16,17}$ and was designated as a cancer registration repository by the local health authority, with compulsory reporting by health care workers. Several years later, cancer registration was mandated to be compulsory by the provincial health authority. In 1974, the QCR started to establish an all cause-of-death certificate reporting system (Qidong All-Death-Cause Registration); This vital statistics system makes available the outcome of any patients with cancer patients who succumbed to the disease or are still alive. The quality of registration data has long been approved by International Agency for Research on Cancer (IARC) and National Central Cancer Registry of China (NCCR). QCR has joined the national monitoring programme (the National Cancer Registration Network) of the NCCR, to provide cancer registration information to Cancer Incidences in Five Continents (CI5) by IARC, to Chinese Cancer Registry Annual Report for many years. ${ }^{18-21}$ For a period of 45 years, the percentage of histology verification (HV) was $15.40 \%(5014 / 32,556)$, with a DCO (death certificate only) rate of $0.25 \%(82 / 32,556) ; 46$ cases were lost of follow up, and 17 died of other diseases or event. The main type of liver cancer was hepatocellular carcinoma (HCC), which accounted for more than $95 \%$ of the liver cancer diagnoses. Cholangiocarcinoma (CC) and other types of liver cancer are not common in this area. All patients with cancer were registered with demographic information; but patients and/or the public were not involved in the design, or conduct, or reporting or dissemination plans of this research.

\section{Follow-Up Methods}

For case follow-up, active method and passive method, or mixed methods were employed, as described elsewhere. ${ }^{13,14}$ Briefly, case-finding lists and death certificate notifications (DCN) were reported monthly. Any cases of mortality from cancer were matched again with the DCN in our registry. For those alive, repeated scrutiny of medical records was performed regularly every year; more careful follow-up was performed again for each 5 -year calendar period. The deadline of last follow up was 31 December 2020. As a result, all new patients or patients who had died that were diagnosed and/or treated in Qidong, or even outside of Qidong, were completely recorded and their outcomes were ascertained. In this paper, all PLC patients were included for analysis.

\section{Survival Analysis}

The main outcome measured in this paper was survival length (period) from the first diagnosis date to the end of follow-up date, or to the death date if one died before the deadline. Cumulative observed survival rate (OS) and relative survival rate (RS) were calculated. RS rate was defined as the ratio of the OS to the expected rate that was 
based on a group of people in the general population similar to the patient group with respects to gender, age and calendar period of observation. The (all-cause) mortality and the life table data of the general population, which contains the probability of death and the life expectancy for single year age-groups for both sexes, for the relevant calendar years, was generated from our Qidong Vital Registry (Qidong All-Death-Cause Registration). The variables concerned were gender ( 1 for male, 2 for female), age group ( 1 for $0-14,2$ for $15-24,3$ for $25-34,4$ for $35-44,5$ for $45-54,6$ for 55-64, 7 for $65-74$, and 8 for 75 and over), and diagnosed period (by 5-year period: 1972-1976, 1977-1981, .., 2007-2011, and 2012-2016; and by 15-year period: 1972-1986, 1987-2001, and 20022016). OS and RS were also calculated by Hakulinen's method; ${ }^{22}$ OS rate and graph were made by the actuarial, or the life-table method, by SPSS Statistics version 22 software; and compared by Wilcoxon (Gehan) statistic. $P<0.05$ was considered as a significant. For trend analysis of RS, the Joinpoint Regression Program ${ }^{23}$ was used to estimate the annual percent change (APC) of RS by sex, and by age group.

\section{Results}

\section{Overall Observed Survival and Relative Survival}

For this 45-year period of liver cancer, 1-, 5-, 10-, 20-, 30-, and 40-year observed survival (OS) rates were 18.51\%, $6.28 \%, 4.03 \%, 2.84 \%, 2.39 \%$, and $1.71 \%$, respectively; their corresponding relative survival (RS) were $18.88 \%$, $6.95 \%, 4.96 \%, 4.49 \%, 5.29 \%$ and $6.11 \%$, respectively. Men had poorer rates than Women did, with 5-year OS of $5.93 \%$, 5-year RS of $6.54 \%$ for males, and of $7.34 \%$ and $8.15 \%$ for females, respectively. Table 1 shows the 5 -year OS and RS rates with 95\% CIs by sex and by calendar year. Figure 1 demonstrates the survival difference in the curve between male and female, with a Gehan statistic of $11.16, P$ less than 0.01 . Figure 2 shows that the APC trends of 5 year RS were increased with the calendar year both for male and female, especially in the years after 2006.

\section{Survival Rate by Age}

Analysis for survival by age-group shows that those at age of 55-64 experienced best survival, with 1-year OS rate of $22.27 \%$, 5-year of $8.44 \%$, and 10 -year of $5.54 \%$; and for $\mathrm{RS}$, these rates were $22.56 \%, 9.09 \%$, and $6.68 \%$, respectively. The elder patients over age 75 had poorer rates of OS and RS (Table 2). There was a significant difference among age-groups, with a Gehan statistic of 214.56, $P$ less than 0.01 (Figure 3). Trend analysis shows that the APCs of 5-year RS for each age group (but age group of 15-24, and of 75+) improved significantly during the 9 periods (Figure 4).

\section{Survival Rate by Period}

Forty-five year data was divided into nine 5-year calendar periods and three 15-year periods. Great improvement in OS and RS could be observed (Table 3 ). For the short-term survival of OS and RS, the recent period of 2002-2016 has showed markedly improvement of $26.56 \%$ in 1 -year OS, compared with the middle period of 1987-2001 (13.02\%) and the early period of 1972-1986 (13.62\%). The 5-year OS has increased from $2.02 \%$ in the early period, $4.40 \%$ in the middle period to $10.76 \%$ in the recent period. Also, significant improvement trend in long-term OS of 10-year could be found: $0.95 \%$ in $1972-1986,3.00 \%$ in $1987-$ 2001, and 7.02\% in 2002-2016 (Table 3). Wilcoxon test shows that the 3 period curves had markedly differences, with a Gehan statistic of $1001.30, P$ value of less than 0.01. Group (period) comparison demonstrates that the recent period had significant high survival than the early and middle periods (Figure 5). For the trends of RSs, Joinpoint Regression shows that the APC of 10-year RS was $5.30 \%$ for the whole period; the APCs of 5-year RS with 2 segments were $3.42 \%$ in $1972-2006$ and $14.64 \%$ in 2006-2016. And for the 1-year RS, 4 segments with APCs of $19.52 \%$ in $1972-1977,-22.34 \%$ in $1977-1981$, and $2.54 \%$ in 1981-2005, and 9.93\% in 2005-2016 (All are significantly different from zero at the alpha $=0.05$ level) (Figure 6).

\section{Discussion}

The survival rate of liver cancer in most clinical data has made great progress in recent years. ${ }^{24}$ Liver resection can be safely performed for multinodular hepatocellular carcinoma (HCC) in the setting of cirrhosis with an overall 5-year survival of $34.6 \%{ }^{25}$ A nationwide multicenter retrospective investigation involving 18,275 out of 42,573 valid HCC patients of hepatectomy from 112 hospitals has showed that the $1,3,5$ years overall survival was $73.2 \%, 28.8 \%$ and $19.6 \%$, respectively. ${ }^{26}$ Shanghai has reported that the 5-year survival rate can reach to $36.2 \%$ for clinical resection cases, and to $64.9 \%$ for small HCC resection cases. The overall 5 year survival rates increased 
Table I Five-Year Observed Survival Rate and Relative Survival Rate of Liver Cancer by Calendar Year in Qidong, 1972-2016 (\%)

\begin{tabular}{|c|c|c|c|c|c|c|c|c|}
\hline \multirow[t]{2}{*}{ Year } & \multicolumn{4}{|c|}{ 5-Year Observed Survival (OS) } & \multicolumn{4}{|c|}{ 5-Year Relative Survival (RS) } \\
\hline & Male & $95 \% \mathrm{Cl}$ & Female & $95 \% \mathrm{Cl}$ & Male & $95 \% \mathrm{Cl}$ & Female & $95 \% \mathrm{Cl}$ \\
\hline 1972 & 0.94 & $0.00-1.88$ & 1.48 & $0.00-3.56$ & 1.02 & $0.01-2.04$ & 1.58 & $0.00-3.80$ \\
\hline 1973 & 0.00 & $\ldots$ & 2.96 & $0.04-5.88$ & 0.00 & $\ldots$ & 3.12 & $0.05-6.19$ \\
\hline 1974 & 3.04 & $1.38-4.70$ & 1.43 & $0.00-3.44$ & 3.28 & I.49-5.07 & 1.55 & $0.00-3.73$ \\
\hline 1975 & 2.66 & $1.26-4.07$ & 6.76 & $2.63-10.88$ & 2.85 & $1.35-4.35$ & 7.30 & $2.84-11.75$ \\
\hline 1976 & 2.63 & $1.13-4.13$ & 4.49 & I. $17-7.80$ & 2.85 & I.23-4.47 & 4.70 & $1.23-8.18$ \\
\hline 1977 & 1.12 & $0.12-2.11$ & 6.90 & $2.69-11.11$ & 1.20 & $0.13-2.26$ & 7.44 & $2.90-11.98$ \\
\hline 1978 & 1.69 & $0.32-3.06$ & 3.48 & $0.06-6.90$ & 1.82 & $0.35-3.30$ & 3.66 & $0.06-7.26$ \\
\hline 1979 & 1.16 & $0.0 I-2.31$ & 0.91 & $0.00-2.72$ & 1.25 & $0.01-2.49$ & 0.98 & $0.00-2.92$ \\
\hline 1980 & 2.37 & $0.8 I-3.93$ & 1.39 & $0.00-3.34$ & 2.57 & $0.88-4.27$ & 1.46 & $0.00-3.51$ \\
\hline 1981 & 0.83 & $0.00-1.79$ & 0.00 & $\ldots$ & 0.91 & $0.00-1.95$ & 0.00 & $\ldots$ \\
\hline 1982 & 0.68 & $0.00-1.46$ & 2.27 & $0.00-4.87$ & 0.74 & $0.00-1.59$ & 2.41 & $0.00-5.17$ \\
\hline 1983 & 1.53 & $0.38-2.68$ & 0.74 & $0.00-2.22$ & 1.66 & $0.42-2.91$ & 0.80 & $0.00-2.38$ \\
\hline 1984 & 2.88 & $|.4|-4.34$ & 2.19 & $0.00-4.69$ & 3.09 & I.52-4.67 & 2.38 & $0.00-5.09$ \\
\hline 1985 & 4.06 & $2.24-5.89$ & 1.44 & $0.00-3.46$ & $4.4 I$ & $2.43-6.39$ & 1.53 & $0.00-3.67$ \\
\hline 1986 & 1.01 & $0.11-1.91$ & 1.41 & $0.00-3.39$ & 1.09 & $0.12-2.06$ & 1.51 & $0.00-3.64$ \\
\hline 1987 & 3.59 & $|.88-5.3|$ & 2.10 & $0.00-4.50$ & 3.85 & $2.02-5.68$ & 2.22 & $0.00-4.76$ \\
\hline 1988 & 4.65 & $2.62-6.68$ & 4.67 & $0.59-8.75$ & 4.98 & $2.8 I-7.16$ & 4.91 & $0.62-9.20$ \\
\hline 1989 & 2.97 & $1.40-4.53$ & 5.43 & I.44-9.42 & 3.17 & $1.50-4.84$ & 5.69 & $|.5|-9.88$ \\
\hline 1990 & 2.24 & $0.90-3.57$ & 5.00 & $1.32-8.68$ & 2.42 & $0.98-3.86$ & 5.30 & I.40-9.21 \\
\hline 1991 & 2.53 & $1.09-3.97$ & 2.17 & $0.00-4.66$ & 2.72 & I. $17-4.27$ & 2.37 & $0.00-5.07$ \\
\hline 1992 & 9.52 & $6.85-12.20$ & 7.58 & $2.97-12.18$ & 10.31 & $7.42-13.20$ & 8.08 & $3.17-12.99$ \\
\hline 1993 & 2.74 & $1.39-4.10$ & 5.08 & $1.95-8.20$ & 3.04 & $1.54-4.53$ & 5.60 & $2.15-9.05$ \\
\hline 1994 & 3.69 & $2.18-5.20$ & 3.98 & $1.22-6.74$ & 4.03 & $2.38-5.69$ & 4.36 & I.34-7.38 \\
\hline 1995 & 3.89 & $2.37-5.42$ & 6.60 & $3.19-10.02$ & 4.32 & $2.62-6.01$ & 7.21 & $3.48-10.93$ \\
\hline 1996 & 4.65 & $2.96-6.33$ & 2.30 & $0.27-4.34$ & 5.23 & $3.33-7.13$ & 2.60 & $0.30-4.91$ \\
\hline 1997 & 4.28 & $2.67-5.89$ & 5.24 & $2.16-8.31$ & 4.83 & $3.01-6.65$ & 5.88 & $2.43-9.33$ \\
\hline 1998 & 4.90 & $3.16-6.65$ & 4.07 & $1.41-6.73$ & 5.49 & $3.53-7.44$ & 4.52 & I.57-7.47 \\
\hline 1999 & 3.83 & $2.33-5.34$ & 4.42 & I.37-7.48 & 4.26 & $2.59-5.92$ & 4.85 & $1.50-8.21$ \\
\hline 2000 & 6.02 & $4.18-7.87$ & 5.75 & $2.65-8.85$ & 6.68 & $4.63-8.72$ & 6.45 & $2.98-9.93$ \\
\hline 2001 & 5.43 & $3.74-7.12$ & 3.18 & $0.62-5.73$ & 6.00 & $4.13-7.87$ & 3.57 & $0.70-6.45$ \\
\hline 2002 & 5.76 & $4.00-7.53$ & 3.93 & $1.02-6.85$ & 6.41 & $4.44-8.37$ & 4.31 & I.I2-7.5I \\
\hline 2003 & 6.27 & $4.35-8.19$ & 6.48 & $3.13-9.83$ & 6.92 & $4.80-9.04$ & 7.26 & $3.5 \mathrm{I}-11.01$ \\
\hline 2004 & 3.79 & $2.36-5.22$ & 6.56 & $3.39-9.73$ & 4.25 & $2.64-5.85$ & 7.53 & 3.89-11.17 \\
\hline 2005 & 5.01 & $3.26-6.76$ & 6.85 & $3.44-10.26$ & 5.65 & $3.67-7.62$ & 7.82 & $3.92-11.72$ \\
\hline 2006 & 6.70 & $4.73-8.67$ & 4.35 & $|.78-6.9|$ & 7.57 & $5.34-9.80$ & 5.11 & $2.09-8.13$ \\
\hline 2007 & 8.13 & $5.97-10.29$ & 9.72 & $6.09-13.35$ & 9.22 & $6.77-11.67$ & 11.48 & $7.19-15.77$ \\
\hline 2008 & 5.85 & $3.99-7.72$ & 6.57 & $3.58-9.56$ & 6.60 & $4.50-8.71$ & 7.60 & $4.14-11.07$ \\
\hline 2009 & 10.41 & $7.98-12.84$ & 10.98 & $7.06-14.90$ & $11.7 \mid$ & $8.99-14.44$ & 12.47 & $8.02-16.91$ \\
\hline 2010 & 11.85 & $9.25-14.46$ & 12.50 & $8.08-16.92$ & 13.20 & $10.30-16.10$ & 14.32 & $9.26-19.38$ \\
\hline 2011 & 11.92 & $9.28-14.56$ & 12.40 & $8.23-16.57$ & 13.28 & $10.34-16.22$ & 13.94 & $9.25-18.62$ \\
\hline 2012 & 9.78 & $7.34-12.22$ & 12.34 & $7.97-16.70$ & 10.97 & $8.24-|3.7|$ & 13.93 & $9.00-18.85$ \\
\hline 2013 & 14.13 & II.19-17.08 & 14.79 & $10.20-19.38$ & $15.9 \mid$ & $\mid 2.59-19.22$ & 17.27 & $11.91-22.63$ \\
\hline 2014 & 15.13 & $12.00-18.27$ & 15.28 & $10.53-20.04$ & 17.38 & $13.78-20.98$ & 18.08 & $12.45-23.70$ \\
\hline 2015 & 18.67 & $15.37-21.97$ & 26.47 & $20.75-32.19$ & 21.26 & $17.50-25.02$ & 30.95 & $24.26-37.64$ \\
\hline 2016 & 24.74 & $20.99-28.49$ & 30.86 & $24.7 I-37.0 I$ & 28.88 & $24.50-33.25$ & 35.36 & $28.3 I-42.4 I$ \\
\hline Total & 5.93 & $5.62-6.23$ & 7.34 & $6.76-7.91$ & 6.54 & $6.21-6.88$ & 8.15 & $7.51-8.79$ \\
\hline
\end{tabular}




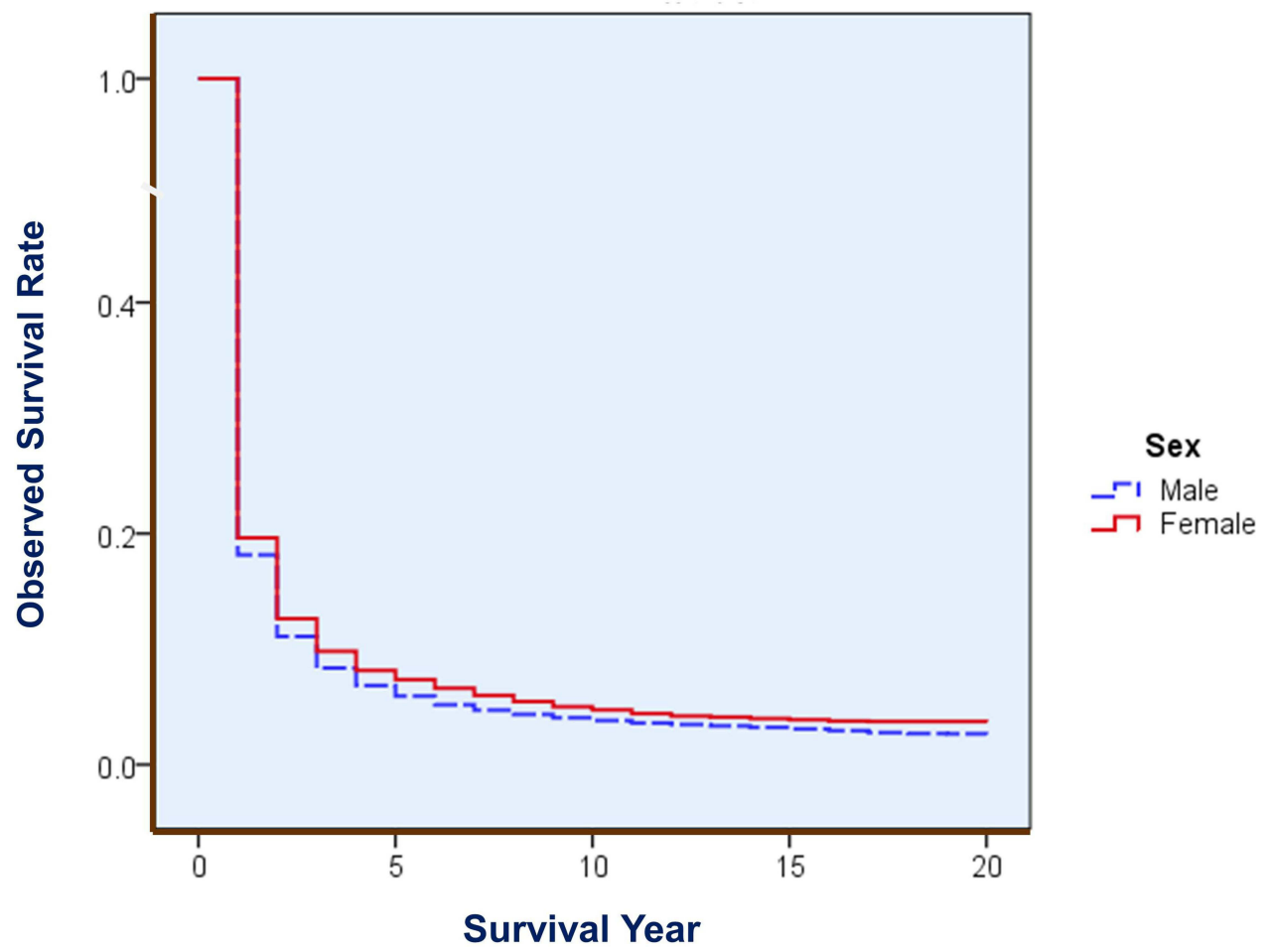

Figure I Overall OS of liver cancer by sex in Qidong, 1972-2016. Gehan statistic is II.156, $P=0.0008$, showing a difference in survival between males and females.

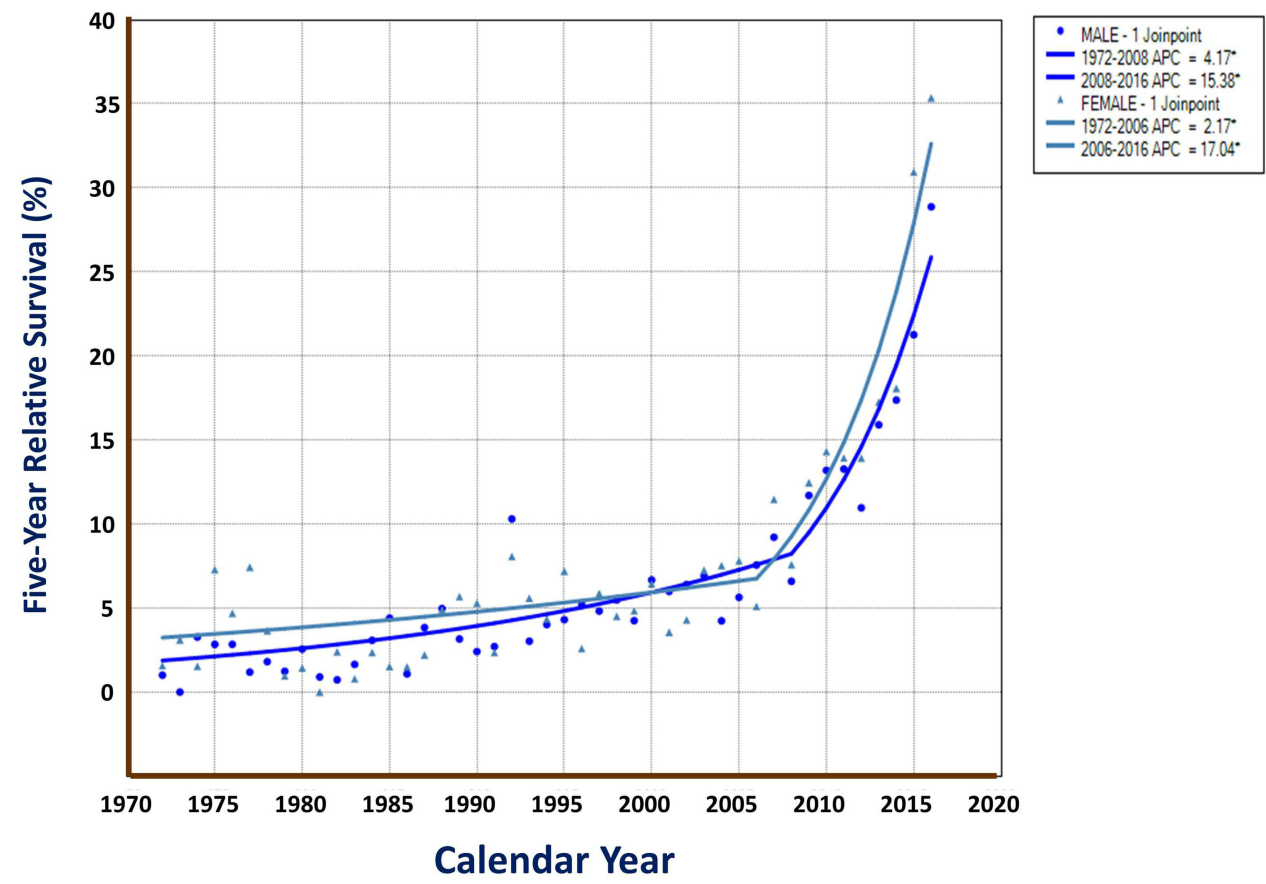

Figure 2 Five-year RS trends of liver cancer by sex in Qidong, 1972-2016. APCs with 2 segments ( 1 joinpoint) were $4.17 \%$ in 1972-2008, and 15.38\% in 2008-2016 in males; and were $2.17 \%$ in 1972-2006, and $17.04 \%$ in 2006-2016 in females (*: P values are less than 0.05 ). 
Table 2 Observed Survival Rate and Relative Survival Rate of Liver Cancer by Age Group in Qidong, 1972-2016 (\%)

\begin{tabular}{|c|c|c|c|c|c|c|c|}
\hline \multirow[t]{2}{*}{ Age Group } & \multirow[t]{2}{*}{$\mathbf{n}$} & \multicolumn{3}{|c|}{ Observed Survival (OS) } & \multicolumn{3}{|c|}{ Relative Survival (RS) } \\
\hline & & $\mathbf{I}-\mathbf{Y r}$ & $5-Y r$ & $10-Y r$ & $\mathrm{I}-\mathbf{Y r}$ & $5-Y r$ & $10-Y r$ \\
\hline $0-14$ & 65 & 20.00 & 6.15 & 4.62 & 20.06 & 6.19 & 4.66 \\
\hline $15-24$ & 317 & 17.35 & 4.73 & 3.79 & 17.37 & 4.76 & 3.84 \\
\hline $25-34$ & 2325 & 14.02 & 4.73 & 3.13 & 14.05 & 4.79 & 3.23 \\
\hline $35-44$ & 7070 & 15.60 & 4.93 & 3.33 & 15.67 & 5.05 & 3.50 \\
\hline $45-54$ & 8723 & 20.13 & 6.79 & 4.61 & 20.27 & 7.04 & 5.02 \\
\hline $55-64$ & 6803 & 22.27 & 8.44 & 5.54 & 22.56 & 9.09 & 6.68 \\
\hline $65-74$ & 4407 & 19.76 & 6.91 & 3.17 & 20.41 & 8.42 & 5.44 \\
\hline $75+$ & 2846 & 13.63 & 3.33 & 1.81 & 15.30 & 6.31 & 7.73 \\
\hline Total & 32,556 & $|8.5|$ & 6.28 & 4.03 & 18.88 & 6.95 & 4.96 \\
\hline
\end{tabular}

from $4.8 \%$ in $1958-1970,12.2 \%$ in $1971-1983$, to $50.5 \%$ in 1984-1996; and for small HCC resection cases, the rates were $33.3 \%, 52.4 \%$ and $67.1 \%$, respectively, in the three periods. ${ }^{27}$ In the view of therapy, surgical resection is the most effective method to improve the survival rate, with high 5 -year rate up to $63 \%$ in Korea, ${ }^{5}$ and even reached to $93.4 \%$ in patients with solitary HCC less than $2 \mathrm{~cm}$ in diameter in Taiwan. ${ }^{28}$ These findings have shown that the level of clinical treatment of liver cancer has indeed improved over the past few decades, among which surgery and small liver cancer have the best therapeutic effects.

However, timely surgical treatment or received other appropriate therapies are not easy to access, which may depend on the degree of early diagnosis for liver cancer. Most of the clinical cases found in outpatient may belong to the middle and advanced stages, and there were very few cases that can get the best surgical treatment. In a clinical study, it showed that the differential stage at diagnosis and treatment were substantially associated

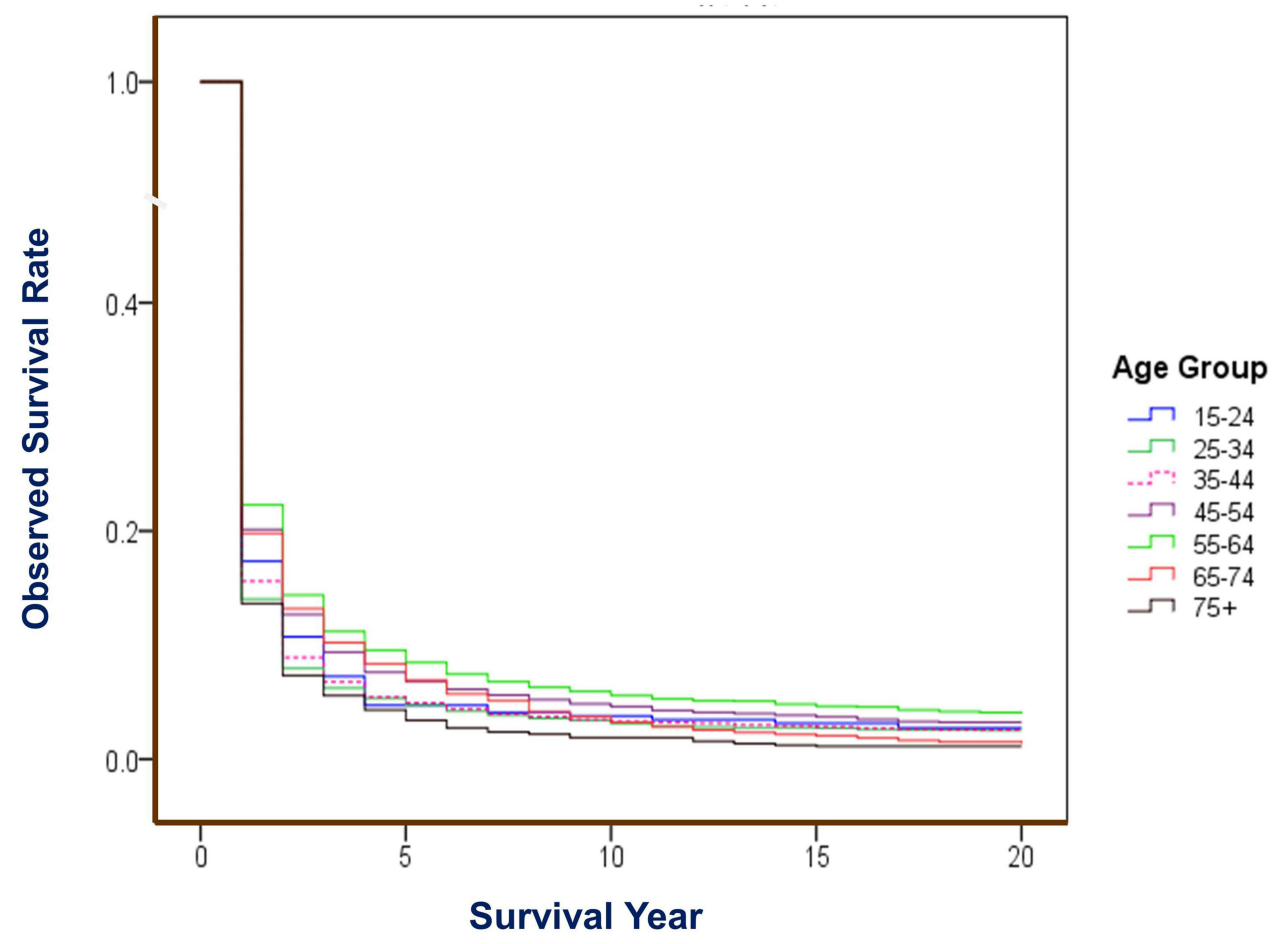

Figure 3 OS of liver cancer by age group in Qidong, 1972-2016. Gehan statistic is $214.561, P=0.0000$, showing differences among age groups (0-14 is not included, due to cases were less than 100). 


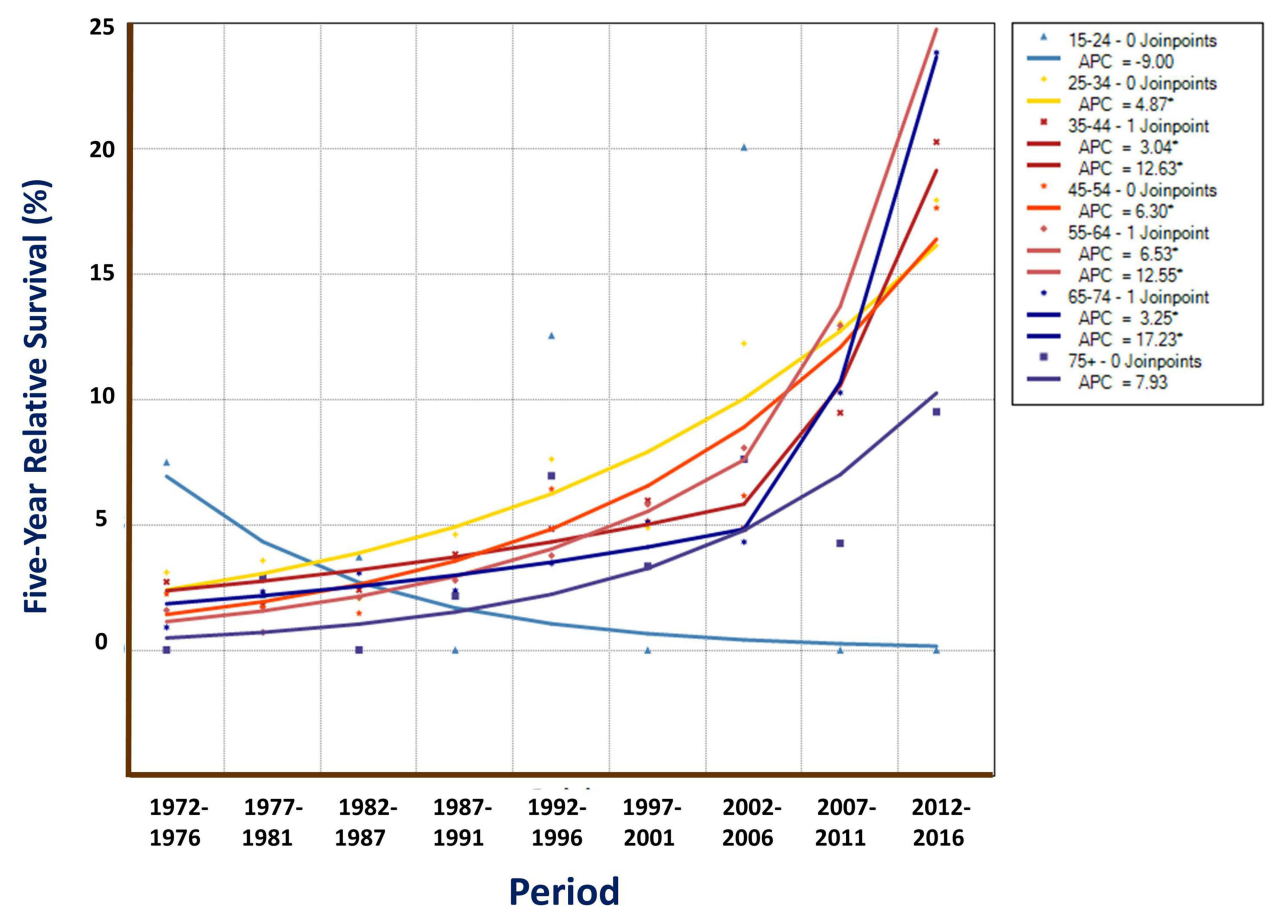

Figure 4 Five-year RS trends of liver cancer by age group in Qidong, 1972-2016. Joinpoint Regression shows that APCs of 5-year RS for those aged at 25-74 were increased markedly $(P s<0.05 *)$, but not significant at age of over 75. Down trend was seen at age group of $15-24$ but $P>0.05$.

with the survival difference, with 1-year survival rates of $71.2 \%, 53.3 \%, 58.3 \%, 27.4 \%$ and $9.4 \%$ for patients at stage IA, IB, II, III, and IV, respectively in $\mathrm{USA}^{29}$ Obviously, if the proportion of early cases is high, the overall survival rate will be high. ${ }^{30}$ Liver cancer screening may be able to detect more early cases, lead to effective early treatment which will benefit the therapeutic effect of liver cancer and improve its survival rate. ${ }^{31-33}$ Therefore, the differences in the survival rates of liver cancer among different countries or regions may reflect the discriminations in the effective treatment of liver cancer as well as the disparities in the early detection of liver cancer. In other words, the improvement in the survival rate of liver cancer in various countries or regions in recent years should be largely affected by the improvement of therapy and the ability of detecting early cases.

A meta-analysis for the results from 63 studies in Asian countries ${ }^{34}$ shows that the 1-, 3- and 5-year survival rate of $\mathrm{HCC}$ were $34.8 \%, 19.0 \%$ and $18.1 \%$, respectively; but it is not all from population-based cancer registries, those with higher survival were mostly from hospital series with relative small sample sizes. ${ }^{35,36}$ In EUROCARE study it is also found that population-based survival for liver cancer was abysmal; the 5-year survival was less than $8 \%$ in European patients with liver cancer, simply due to the potentially curative techniques and transarterial chemoembolization can be utilized in a low percentage of cases, with a consequent little impact on populationbased survival. ${ }^{37}$

The prognosis of liver cancer from population-based registered cases is actually an embodiment of this capacity of comprehensive treatment and health service, so it is different from the results of a certain therapy or the treatment capacity of a certain hospital. The EUROCARE studies or CONCORD series research have provided the world a large number of results from population-based registration, ${ }^{11,12}$ but each of these studies basically reflected the sectional results of certain periods by country or region, and may not deduce the historical changes (trends). In this study, based on the long-run collection of all liver cancer cases from all residents in a coverage area of a population-based cancer registry in China, the characteristics and changes of liver cancer survival rates in a 45-year period is analyzed, providing an example of realworld profile for the estimation of long-term trends of liver cancer survival.

The area of QCR covered was a typical rural county (although after 1989 renamed as a city) in Jiangsu Province, China, located in the eastern coastal areas, with about $1.10 \mathrm{M}-1.16 \mathrm{M}$ population during the decades. The level of medical service was among the 
Table 3 Observed Survival Rate and Relative Survival Rate of Liver Cancer by Period in Qidong, 1972-2016 (\%)

\begin{tabular}{|c|c|c|c|c|c|c|c|}
\hline \multirow[t]{2}{*}{ Period } & \multirow[t]{2}{*}{$\mathbf{n}$} & \multicolumn{3}{|c|}{ Observed Survival (OS) } & \multicolumn{3}{|c|}{ Relative Survival (RS) } \\
\hline & & $\mathrm{I}-\mathrm{Yr}$ & $5-Y r$ & $10-Y r$ & $\mathrm{I}-\mathrm{Yr}$ & $5-Y r$ & $10-Y r$ \\
\hline $1972-1976$ & 2937 & 16.11 & 2.32 & 0.95 & 16.32 & 2.49 & 1.13 \\
\hline |977-|98| & 2503 & 15.46 & 1.76 & 0.88 & 15.66 & 1.89 & 1.04 \\
\hline $1982-1986$ & 3067 & 9.75 & 1.96 & 1.01 & 9.88 & 2.11 & 1.20 \\
\hline |987-|99| & 2999 & 11.04 & 3.30 & 2.43 & 11.18 & 3.54 & 2.86 \\
\hline $1992-1996$ & 3915 & 13.05 & 4.75 & 3.58 & 13.29 & 5.24 & 4.41 \\
\hline |997-200| & 4304 & 14.36 & 4.83 & 2.86 & 14.68 & 5.38 & 3.55 \\
\hline 2002-2006 & 4415 & $|6.5|$ & 5.53 & 3.31 & 16.88 & 6.21 & 4.17 \\
\hline 2007-20II & 4396 & 25.32 & 9.80 & 6.52 & 26.01 & 11.08 & 8.28 \\
\hline $2012-2016$ & 4020 & 38.96 & 17.61 & $\ldots$ & 39.99 & 20.17 & $\ldots$ \\
\hline $1972-1986$ & 8507 & 13.62 & 2.02 & 0.95 & 13.81 & 2.18 & 1.13 \\
\hline |987-200| & 11,218 & 13.02 & 4.40 & 3.00 & 13.25 & 4.83 & 3.65 \\
\hline $2002-2016$ & $|2,83|$ & 26.56 & 10.76 & 7.02 & 27.24 & 12.18 & 8.96 \\
\hline $1972-2016$ & 32,556 & $|8.5|$ & 6.28 & 4.03 & 18.88 & 6.95 & 4.96 \\
\hline
\end{tabular}

middle-degrees in China, but the research on prevention and treatment of liver cancer had started earlier since 1970's. We have reported that the crude mortality rate of liver cancer in this area had experienced trends from lower to higher levels, and from continued increase at a high plateau to most recently a gradual decline. ${ }^{17} \mathrm{We}$ believe that many years of comprehensive prevention and intervention measures must have not only influenced the decline of the liver cancer incidence/mortality, but also the survival of the liver cancer patients from the population of this area. Table 1 has showed that the 5-year observed survival (OS) and relative survival (RS) rates during the early calendar years were poor, but improved during the recent years (Figure 2). Women experienced relative high OS and RS rates than men did (Figure 1). Table 2 and Figure 3 demonstrate that there were significant differences in the survival rates among age groups, with better prognosis at age of 5564, poor results at aged over 75. Joinpoint Regression shows that the trends of 5-year RS for each age group were increased markedly, but not for the younger aged at 15-24 (Figure 4), since the most younger patients were occurred in the early period ${ }^{17}$ when the survival rates were lower as Table 1 showed.
Generally, the survival rates in young and middle-aged patients were higher, while the survival rate in elderly patients was relatively poor. An analysis of 27,255 patients derived from SEER database has showed that the 5-year liver cancer survival rates were higher in the younger age groups than in the older age groups, with rates of $14.5 \%$ vs $8.4 \%{ }^{38}$ Although the overall survival rate in our area was lower, when compared the 5-year rates for the early, middle and recent periods, we would find, from Table 3 and Figure 5, that the survival rates have been improved greatly, for 5-year OS, from $2.02 \%$ in $1972-1986$, to $4.40 \%$ in $1987-2001$, and to $10.76 \%$ in $2002-2016$; and for 5 -year RS, from $2.18 \%$, to $4.83 \%$, and to $12.18 \%$, respectively.

The overall survival rate of patients with liver cancer from a covered area may be affected by many factors, ${ }^{13}$ but mostly, by factors such as the proportion of early cases (the proportion of patients found in screening), the proportion of patients received surgery, and the proportion of patients treated with appropriate therapies, or even by the hospital volume that is associated with improved survival for both surgical and non-surgical patients with liver cancer. ${ }^{8}$ In Qidong's practice, surgical operation was the best way of improving prognosis of liver cancer; during 


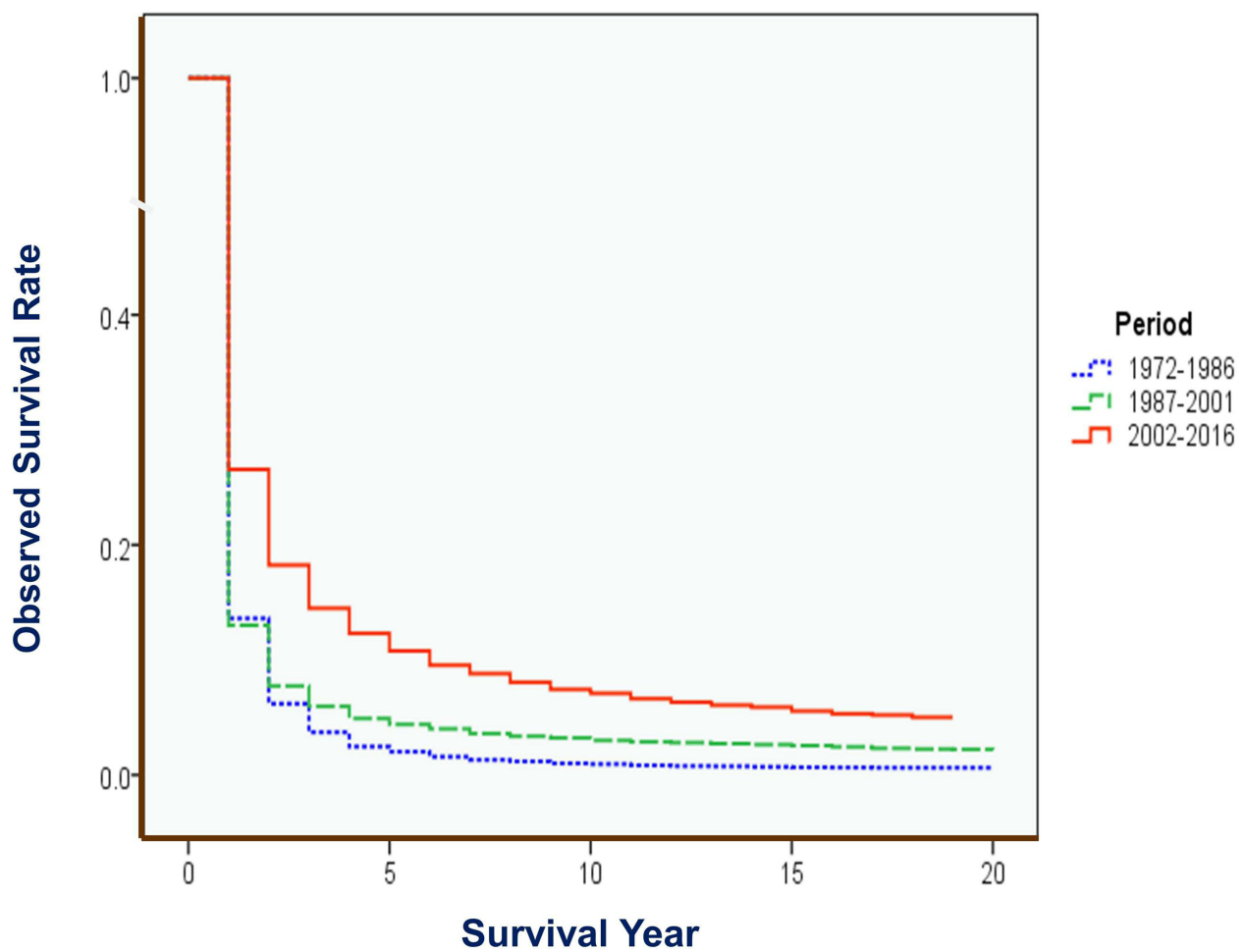

Figure 5 OS of liver cancer by 15-year period in Qidong, 1972-2016. Gehan statistic of total test is 1001.303, $P=0.0000$. Group comparison [period testing]: Early period (blue) vs Middle period (green): $0.181, P=0.6705$; Early period vs Recent period (red): $599.730, P=0.0000$; Middle period vs Recent period: $707.413, P=0.0000$.
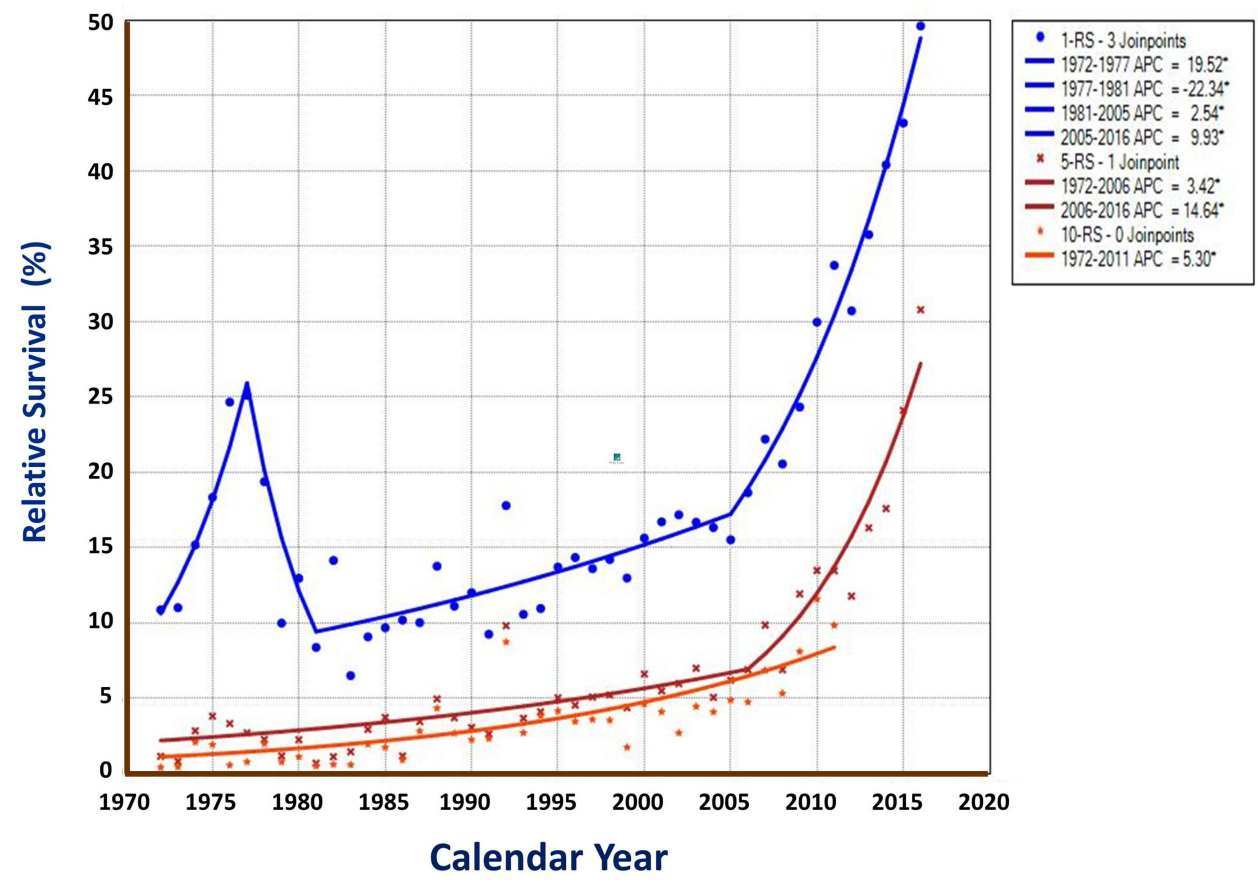

Figure 6 RS trends of liver cancer by survival year in Qidong, 1972-2016. Joinpoint Regression shows that the APC of 10-year RS, the APCs of 5-year RS with 2 segments, and APCs of I-year RS with 4 segments were all significantly different from zero at the level of alpha $=0.05^{*}$. 
the period of 1972-1984, 1-, 3-, and 5-year survival rates could be up to $77.3 \%, 39.3 \%$ and $34.6 \%$ respectively. ${ }^{39}$ In Figure 6, the 1-year RS trend has showed a very interesting "pulse" increase, this could be explained by the alphafetoprotein (AFP) mass screening of over $1.8 \mathrm{M}$ person times during the middle 1970's which resulted in a proportion of $35 \%$ early state cases with PLC. ${ }^{33}$ The 5-year and 10-year RS trends may be the real reflection of the improved survival during the long-term period.

A recent study also showed that screening (with semiannual AFP and B ultrasound) individuals at high-risk of development of liver cancer after 2006 is effective not only in increasing detection rate but also in detecting liver cancer at early stage, and in improving patients' survival as well, with 1-, 3-, 5-year survival rates of $77.16 \%$, $49.04 \%$, and $38.53 \%$ in the repeat periodic screening group, ${ }^{32}$ implying that the improvement in survival over the last decade shown in Figure 6 may be due to early detection and effective treatment. An Australian prospective population-based study showed that increased survival was associated with participation in surveillance programs and curative treatment. The 1-, and 2-year survival rates for surveillance participants were 79\% and $66 \%$, compared with $49 \%$ and $33 \%$ for nonparticipants, respectively. ${ }^{40}$ Therefore, we can conclude that screening and early appropriate treatment are key factors in improving the survival rate of liver cancer, but if these cases account for a relatively small proportion of patients in the entire coverage area, the overall survival rate may not be very high, even if the single therapy is effective. $^{39}$

There are some limitations of this study. First, the proportion of histology verification (HV\%) in cases was not very high although most patients were diagnosed with AFP and or B ultrasound/CT scan. Second, we have known that early stage would be benefit to the survival, but we could not collect the detailed information on the TNM classification from our population-based cancer registration. Third, epidemiological evidence has revealed that liver cancer or HCC is associated with hepatitis B virus (HBV), but we did not analyze survival by HBV status. However, most patients with liver cancer were HBV-related cases in Qidong area, as indicated in an investigation $^{41}$ that $\mathrm{HBV}$-related cases reached up to $100 \%(181 / 181)$, and only a few (6/181) were coinfected with hepatitis $\mathrm{C}$ virus.

Data from cancer registries and linked treatment records can provide unique insights into outcomes in routine oncology practice, by asking the right questions with the right data and the right methods. Real world data has the potential to close gaps in our knowledge of cancer and to identify gaps between evidence and practice that will ultimately lead to improved patient care and outcomes. ${ }^{15}$ Our paper reflects the real world observation results of the 45 year survival rate of liver cancer in all the population in Qidong, China, showing that great improvement has been achieved over the past decades. This imply that in order to improve the overall survival rate of liver cancer in a region, not only the level of clinical treatment, but also the coverage of appropriate medical services and the ability of finding more early cases should be improved, depending on joint efforts of clinicians or researchers and public health personnel in the area.

\section{Abbreviations}

PLC, Primary liver cancer; RWD, Real world data; QCR, Qidong Cancer Registry; CI5, Cancer Incidences in Five Continents; IARC, International Agency for Research on Cancer; HV, Histology verification; DCO, Death certificate only; DCN, Death certificate notifications; OS, Observed survival; RS, Relative survival; 95\% CI, 95\% confidence interval; HCC, Hepatocellular carcinoma; AFP, Alphafetoprotein; HBV, hepatitis B virus.

\section{Patient and Public Involvement}

Patients and/or the public were not involved in the design, or conduct, or reporting, or dissemination plans of this research.

\section{Data Sharing Statement}

Data are available upon request from the correspondence author, Prof. Jian-Guo Chen.

\section{Ethics Approval}

The ethical committee (IRB) of Qidong Liver Cancer Institute/Qidong People's Hospital approved the study.

\section{Acknowledgments}

This work was supported by Qidong Cancer Registry of Qidong Liver Cancer Institute which collects the data reported by the health workers in the Qidong Health Care System. We thank the staff of the 12 towns in Qidong for undertaking the case-finding and follow-up. 


\section{Author Contributions}

All authors made a significant contribution to the work reported, whether that is in the conception, study design, execution, acquisition of data, analysis and interpretation, or in all these areas; took part in drafting, revising or critically reviewing the article; gave final approval of the version to be published; have agreed on the journal to which the article has been submitted; and agree to be accountable for all aspects of the work. All authors have individually approved the final version of this manuscript.

\section{Disclosure}

The authors report no conflicts of interest in this work.

\section{References}

1. Sung H, Ferlay J, Siegel RL, et al. Global cancer statistics 2020: GLOBOCAN estimates of incidence and mortality worldwide for 36 cancers in 185 countries. CA Cancer J Clin. 2021;71(3):209-249. PMID: 33538338. doi:10.3322/caac. 21660

2. Zhang SW, Sun KX, Zheng RS, et al. Cancer incidence and mortality in China, 2015. J Natl Cancer Center. 2020;1(1):2-11. doi:10.1016/j. jncc.2020.12.001

3. Chen JG, Zhang SW. Liver cancer epidemic in China: past, present and future. Semin Cancer Biol. 2011;21(1):59-69. PMID: 21144900. doi:10.1016/j.semcancer.2010.11.002

4. Rich NE, Yopp AC, Singal AG. Medical management of hepatocellular carcinoma. $J$ Oncol Pract. 2017;13(6):356-364. PMID: 28605614. doi:10.1200/JOP.2017.022996

5. Kim H, Ahn SW, Hong SK, et al. Survival benefit of liver resection for Barcelona Clinic Liver Cancer stage B hepatocellular carcinoma. Br J Surg. 2017;104(8):1045-1052. PMID: 28480964. doi:10.1002 bjs. 10541

6. Xia Y, Li J, Liu G, et al. Long-term effects of repeat hepatectomy vs percutaneous radiofrequency ablation among patients with recurren hepatocellular carcinoma: a randomized clinical trial. JAMA Oncol. 2020;6(2):255-263. PMID: 31774468; PMCID: PMC6902111. doi:10.1001/jamaoncol.2019.4477

7. Linn YL, Chee MY, Koh YX, et al. Actual 10-year survivors and 10 -year recurrence free survivors after primary liver resection for hepatocellular carcinoma in the 21st century: a single institution contemporary experience. J Surg Oncol. 2021;123(1):214-221. PMID: 33095920. doi:10.1002/jso.26259

8. Mokdad AA, Zhu H, Marrero JA, et al. Hospital volume and survival after hepatocellular carcinoma diagnosis. $\mathrm{Am}$ $J$ Gastroenterol. 2016;111(7):967-975. PMID: 27166130. doi:10.1038/ajg.2016.181

9. Chen JG, Chen HZ, Zhu J, et al. Cancer survival in patients from a hospital-based cancer registry, China. J Cancer. 2018;9(5):851-860. PMID: 29581763; PMCID: PMC5868149. doi:10.7150/jca.23039

10. Are C, Meyer B, Stack A, et al. Global trends in the burden of liver cancer. J Surg Oncol. 2017;115(5):591-602. PMID: 28345140. doi: $10.1002 /$ jso. 24518

11. Allemani C, Matsuda T, Di Carlo V, et al. Global surveillance of trends in cancer survival 2000-14 (CONCORD-3): analysis of individual records for 37513025 patients diagnosed with one of 18 cancers from 322 population-based registries in 71 countries. Lancet. 2018;391(10125):1023-1075. PMID: 29395269; PMCID: PMC5879496. doi:10.1016/S0140-6736(17)33326-3
12. Dal Maso L, Panato C, Tavilla A, et al. Cancer cure for 32 cancer types: results from the EUROCARE-5 study. Int $J$ Epidemiol. 2020;49(5):1517-1525. PMID: 32984907. doi:10.1093/ije/dyaa128

13. Sankaranarayanan R, Black R, Swaminathan R, eds. Cancer Survival in Developing Countries. IARC Sci Publ No. 145. Lyon: IARC; 1998:1-173.

14. Sankaranarayanan R, Swaminathan R, eds. Cancer Survival in Africa, Asia, the Caribbean and Central America. IARC Sci Publ No. 162. Lyon: IARC; 2011:1-291.

15. Booth CM, Karim S, Mackillop WJ. Real-world data: towards achieving the achievable in cancer care. Nat Rev Clin Oncol. 2019;16(5):312-325. PMID: 30700859. doi:10.1038/s41571-0190167-7

16. Chen JG, Zhu J, Parkin DM, et al. Trends in the incidence of cancer in Qidong, China, 1978-2002. Int J Cancer. 2006;119:1447-1454. doi:10.1002/ijc. 21952

17. Chen JG, Zhu J, Zhang YH, et al. Liver cancer mortality over six decades in an epidemic area: what we have learned. PeerJ. 2021;9: e10600. PMID: 33604165; PMCID: PMC7866902. doi:10.7717/ peerj. 10600

18. Forman D, Bray F, Brewster DH, eds. Cancer Incidence in Five Continents. Vol. X. IARC Sci Publ No. 164. Lyon: IARC; 2013:1-1365.

19. Chen W, Zheng R, Baade PD, et al. Cancer statistics in China, 2015. CA Cancer J Clin. 2016;66:115-132. doi:10.3322/caac.21338

20. National Cancer Center. Chinese Cancer Registry Annual Report 2016. Beijing: Tsinghua University Publishing House; 2016:1-550.

21. Bray F, Colombet M, Mery L, eds. Cancer Incidence in Five Continents. Vol. XI. IARC Sci Publ No. 166. Lyon: IARC; 2021:550-551.

22. Hakulinen T, Abeywickrama K. A computer program package for relative survival analysis. Comput Programs Biomed. 1985;19:197-207. doi:10.1016/0010-468X(85)90011-X

23. National Cancer Institute. Joinpoint Regression Program, Version 4.7.0.0. Bethesda, MD: National Cancer Institute; February, 2019. Available from: http://surveillance.cancer.gov/joinpoint/. Accessed March 23, 2020.

24. Koulouris A, Tsagkaris C, Spyrou V, et al. Hepatocellular carcinoma: an overview of the changing landscape of treatment options. J Hepatocell Carcinoma. 2021;8:387-401. doi:10.2147/JHC.S300182

25. Li ZL, Yu JJ, Guo JW, et al. Liver resection is justified for multinodular hepatocellular carcinoma in selected patients with cirrhosis: a multicenter analysis of 1066 patients. Eur J Surg Oncol. 2019;45 (5):800-807. PMID: 30594407. doi:10.1016/j.ejso.2018.12.016

26. Zhang B, Zhang B, Zhang Z, et al. 42,573 cases of hepatectomy in China: a multicenter retrospective investigation. Sci China Life Sci. 2018;61(6):660-670. PMID: 29417360. doi:10.1007/s11427-0179259-9

27. Tang ZY, Zhou XD, Ma ZC, et al. Multimodality treatment of hepatocellular carcinoma. J Gastroenterol Hepatol. 1998;13(S3): S315-S319. PMID: 28976647. doi:10.1111/j.1440-1746.1998. tb01901.X

28. Hsiao CY, Hu RH, Ho CM, et al. Surgical resection versus radiofrequency ablation for Barcelona Clinic Liver Cancer very early stage hepatocellular carcinoma: long-term results of a single-center study. Am J Surg. 2020;220(4):958-964. PMID: 32247523. doi:10.1016/j. amjsurg.2020.03.017

29. Lin YJ, Lin CN, Sedghi T, et al. Treatment patterns and survival in hepatocellular carcinoma in the United States and Taiwan. PLoS One. 2020;15(10):e0240542. PMID: 33052942; PMCID: PMC7556438. doi:10.1371/journal.pone. 0240542

30. Chen JG, Parkin DM, Chen QG, et al. Screening for liver cancer: results of a randomised controlled trial in Qidong, China. $J$ Med Screen. 2003;10(4):204-209. PMID: 14738659. doi:10.1258/ 096914103771773320 
31. Sarkar M, Stewart S, Yu A, et al. Hepatocellular carcinoma screening practices and impact on survival among hepatitis B-infected Asian Americans. J Viral Hepat. 2012;19:594-600. doi:10.1111/j.13652893.2011.01577.x

32. Chen JG, Zhang YH, Zhu J, et al. Early diagnosis and early treatment for liver cancer in Qidong: survival of patients and effectiveness of screening. Chin J Oncol. 2017;39(12):946-951. PMID: 29262514. doi:10.3760/cma.j.issn.0253-3766.2017.12.013

33. Chen JG, Zhang YH, Lu LL, et al. Liver cancer screening in China: practices and its extended questions. Hepatoma Res. 2019;5:2. doi:10.20517/2394-5079.2019.03

34. Hassanipour S, Vali M, Gaffari-Fam S, et al. The survival rate of hepatocellular carcinoma in Asian countries: a systematic review and meta-analysis. EXCLI J. 2020;19:108-130. PMID: 32038120; PMCID: PMC7003639. doi:10.17179/excli2019-1842

35. Toyoda H, Kumada T, Kiriyama S, et al. Changes in the characteristics and survival rate of hepatocellular carcinoma from 1976 to 2000: analysis of 1365 patients in a single institution in Japan. Cancer. 2004;100:2415-2421. doi:10.1002/cncr.20289

36. Zhang Y, Li Y, Cai J. Surgical management of patients with BCLC b hepatocellular carcinoma and survival analysis: a single center experience in China. Eur J Cancer. 2015;51:S425. doi:10.1016/ S0959-8049(16)31194-7
37. Capocaccia R, Sant M, Berrino F, et al. Hepatocellular carcinoma: trends of incidence and survival in Europe and the United States at the end of the 20th century. Am J Gastroenterol. 2007;102(8):16611670;quiz 1660, 1671. PMID: 17555459. doi:10.1111/j.15720241.2007.01337.x

38. Zhang W, Sun B. Impact of age on the survival of patients with liver cancer: an analysis of 27,255 patients in the SEER database. Oncotarget. 2015;6(2):633-641. PMID: 25575810; PMCID: PMC4359244. doi:10.18632/oncotarget.2719

39. Zhang BC, Wang MR, Huang XY, et al. Clinical study of subclinical hepatic cancer: analysis of 187 patients. Chin J Oncol. 1987;9 (6):451-453.

40. Hong TP, Gow PJ, Fink M, et al. Surveillance improves survival of patients with hepatocellular carcinoma: a prospective population-based study. Med J Aust. 2018;209:348-354. doi:10.5694/mja18.00373

41. Ming L, Thorgeirsson SS, Gail MH, et al. Dominant role of hepatitis $\mathrm{B}$ virus and cofactor role of aflatoxin in hepatocarcinogenesis in Qidong, China. Hepatology. 2002;36(5):1214-1220. doi:10.1053/ jhep.2002.36366
Journal of Hepatocellular Carcinoma

\section{Publish your work in this journal}

The Journal of Hepatocellular Carcinoma is an international, peerreviewed, open access journal that offers a platform for the dissemination and study of clinical, translational and basic research findings in this rapidly developing field. Development in areas including, but not limited to, epidemiology, vaccination, hepatitis therapy, pathology

\section{Dovepress}

and molecular tumor classification and prognostication are all considered for publication. The manuscript management system is completely online and includes a very quick and fair peer-review system, which is all easy to use. Visit http://www.dovepress.com/ testimonials.php to read real quotes from published authors. 